Dialectologia 24 (2020), 91-109.

ISSN: 2013-2247

Received 30 January 2018.

Accepted 3 May 2018.

\title{
EL VOSEO EN MEDELLÍN, COLOMBIA: \\ UN RASGO DIALECTAL DISTINTIVO DE LA IDENTIDAD PAISA
}

\author{
Diana FERNÁNDEZ ACOSTA \\ Western University * \\ dferna52@uwo.edu
}

\section{Resumen}

En este estudio se investigó el fenómeno del voseo en Medellín, Colombia para establecer qué factores influyen en su uso y determinar si los hablantes relacionan esta característica dialectal con la identidad de la cultura paisa. Diez habitantes de Medellín de clases alta y media alta suministraron información a través de cuestionarios. Los datos recogidos muestran que el tipo de relación con los interlocutores, su edad y el género de los hablantes son factores que influyen en el uso de vos como forma de tratamiento. El análisis de los resultados también muestra que los paisas, sin importar su edad, ven el voseo como parte de su identidad y como un fenómeno lingüístico que les da pertenencia a su grupo regional. Este estudio constituye la base para futuros estudios sobre el fenómeno y aporta una reflexión al campo de la enseñanza sobre la importancia de incluir el voseo en las aulas de español como lengua extranjera.

\section{Palabras clave}

voseo, cultura paisa, formas de tratamiento

\footnotetext{
* Western University, 1151 Richmond Street, London, Ontario, Canada, N6A 3K7.
} 


\section{VOSEO IN MEDELLIN, COLOMBIA: A DISTINCTIVE DIALECTAL FEATURE OF PAISA IDENTITY}

\section{Abstract}

This study investigated the voseo in Medellin, Colombia to determine which factors contribute to its use and whether or not speakers consider this dialectal feature to be part of the paisa culture. Ten upper class speakers from Medellin provided information through questionnaires. Data show that the kind of relationship with interlocutors, their age, and the gender of the speakers are factors that influence the use of vos as a form of address. The analysis of the results shows that paisas, regardless of their age, see the voseo as part of their identity and as a linguistic phenomenon of membership to their community. This study constitutes the basis for future studies about this phenomenon and a point of reflection in the teaching field about the importance of including voseo in L2 Spanish classrooms.

\section{Keywords}

voseo, paisa culture, forms of address

\section{Introducción}

El voseo es una forma de tratamiento pronominal que utiliza el vos singular en lugar de tú para dirigirse a un solo interlocutor. Ocupa la tercera parte del continente americano y es uno de los fenómenos morfosintácticos dialectales más interesantes en la variedad del español de América. El voseo figura en Argentina, Costa Rica, Chile, Honduras, Guatemala, Nicaragua, el Salvador y en partes de Paraguay, Uruguay, Bolivia y Colombia. (Angulo 2009; Benavides 2003; Kapovic 2007). Existen tres formas de voseo como puede verse en la Tabla 1: (1) el voseo pleno donde el pronombre vos va acompañado de las formas verbales correspondientes a vos, (2) el voseo pronominal que utiliza el pronombre vos con conjugaciones de tú y (3) el voseo verbal donde el pronombre tú va acompañado de conjugaciones de vos. 


\begin{tabular}{|c|c|c|c|}
\hline & Uso del pronombre & $\begin{array}{c}\text { Uso de la forma } \\
\text { verbal }\end{array}$ & Ejemplo \\
\hline $\begin{array}{c}\text { Voseo pleno o } \\
\text { auténtico }\end{array}$ & sí & sí & vos comés \\
\hline Voseo pronominal & sí & no & vos comes \\
\hline Voseo verbal & no & sí & comés \\
\hline
\end{tabular}

Tabla 1. Tipología del voseo.

Villegas (1963) explica que el pronombre vos puede ser evidenciado en obras literarias incluso tan antiguas como El Cantar de Mio Cid que data del siglo trece y en cuyas páginas se puede ver cómo se usa el vos como forma respetuosa de tratamiento a personas importantes mientras que tú es utilizado para referirse a personas de rango inferior. Así mismo, en España en los siglos dieciséis y diecisiete se usaba el voseo para el trato respetuoso a personas distinguidas de la alta sociedad y fue así como en la época de la conquista los españoles trajeron el voseo a América con la intención, tal vez, de hacerse sentir importantes en nuevas tierras (Angulo 2009). En el siglo XVIII, por razones poco conocidas, el voseo en España comenzó a ser asociado con clases sociales bajas lo que llevó a los habitantes españoles a descontinuar su uso progresivamente y así vos fue remplazado casi en su totalidad por tú para tratamiento familiar y por el uso de vuestra merced como forma de tratamiento respetuoso. Estos nuevos cambios de pérdida de prestigio en el uso del voseo llegaron también a América, aunque solo a las regiones que tenían mayor flujo de inmigrantes españoles y mayor contacto con centros administrativos y culturales de la colonia. Las regiones más apartadas y marginadas de los usos lingüísticos peninsulares no participaron de estos cambios dado su aislamiento económico, político y cultural (Benavides 2003). En estas regiones, como es el caso de Medellín, el voseo continuó siendo la norma estándar y socialmente aceptada y que predomina en la actualidad.

El voseo es un fenómeno relativamente poco estudiado. Es decir, se ha escrito mucho sobre el tema de manera descriptiva pero no existe una cantidad considerable de estudios sobre él. Esto es especialmente cierto para Colombia, un país con un voseo 
regional en vez de nacional que pasa casi desapercibido en estudios sobre este fenómeno que prefieren enfocarse en otros países con voseo generalizado como Argentina o Costa Rica. El valor dialectal y sociolingüístico de este estudio radica en el análisis que hace del voseo en un contexto más específico al enfocarse en una ciudad donde las tres formas de tratamiento pronominal -tú, usted y vos-conviven día a día.

\subsection{Preguntas de Investigación}

El presente estudio tiene dos preguntas de investigación, a saber:

(1) ¿Cuáles son los factores que delimitan el uso del voseo en el habla de los habitantes de Medellín? Se examinaron específicamente la edad del interlocutor, la relación con el interlocutor y el género de los hablantes.

(2) ¿Tienen los hablantes paisas una actitud de identidad regional y de pertenencia a su grupo social que motiva el uso del vos? ¿Tiene la edad de los hablantes algún efecto en esta actitud?

\section{Revisión de la bibliografía}

Como ya se ha mencionado, el cambio de vos a tú que se generó en España a causa de la pérdida de prestigio del voseo llegó y se estableció también en América, pero solo en los lugares más conectados con la corona por motivos comerciales y de movilidad. En el caso específico de Colombia, el virreinato establecido por España en el siglo XVIII tuvo sede en la capital, Bogotá y fue esta ciudad la que primero adoptó las normas lingüísticas peninsulares. Este no fue el caso de Medellín, sin embargo, cuya geografía altamente montañosa dificultaba enormemente la comunicación y el transporte en tiempos coloniales generando un aislamiento entre las personas que allí se habían establecido y el resto del país. Por tal razón, los habitantes de Medellín de aquella época se acostumbraron a una realidad de independencia que creó conceptos de identidad cultural en la región tales como el mito de la antioqueñidad que es el modelo mental que los 
paisas tienen de sí mismos como una raza emprendedora y pujante. Esta comunidad que se desarrolló aislada en las montañas adoptó costumbres diferenciadas como la religiosidad, los valores familiares cimentados en una gran responsabilidad para con la familia y la voluntad del trabajo constante. Al respecto, Millán (2011) afirma que el área de Antioquia es caracterizada por un intenso uso del vos en todas las clases sociales y contextos y que esto puede deberse al tipo de sociedad igualitaria y abierta que allí se formó en la época de la colonia ya que no existía en la región una población indígena numerosa que pudiera proveer una clase servil y así todos tuvieron que igualarse en el trabajo y mantuvieron el vos como forma de confianza. En su estudio del voseo en Cali y Medellín con estudiantes de universidad, encontró que factores no lingüísticos tales como el sexo, la clase social, la edad, la relación con el interlocutor y el lugar de interacción influyen en el uso de vos y que vos es utilizado mayormente en situaciones de extrema confianza e intimidad con el interlocutor y con personas de la misma edad o más jóvenes. También encontró que los pronombres vos, tú y usted están socialmente marcados. Según sus resultados, el uso de vos y tú incrementa mientras incrementa la clase social y usted es más utilizado por hablantes de clases sociales más bajas. Otro hallazgo importante del estudio de Millán es que hay más variedad en el habla de las mujeres y que ellas presentan una tendencia mayor a utilizar tú que los hombres. Atribuye este hallazgo al hecho ya conocido de que son las mujeres las que tienden a usar formas de prestigio más frecuentemente que los hombres y, por ser el tuteo la norma en la capital del país, es la forma de tratamiento que es considerada estándar.

Jang (2015), por otro lado, dice que las mujeres tienden a utilizar más el tuteo que los hombres porque éste tiene un matiz feminizante. En su estudio con estudiantes de Medellín, encontró que el tú puede sonar femenino cuando se emplea entre interlocutores masculinos y, por ende, esta forma de tratamiento es más utilizada por las mujeres. Agrega que el tuteo se considera una forma de tratamiento no propia de la región antioqueña, una forma relativamente nueva comparada con el vos. Sin embargo, parece estar extendiéndose en la zona poco a poco. Esto no significa que el tuteo va a remplazar a voseo en Medellín, por lo menos no en el corto plazo, ya que "el voseo forma parte de la identidad regional de los antioqueños" (Jang 2015: 85). Frente a la incógnita 
de si el voseo en Medellín tiende a desaparecer con el tiempo, algunos autores dicen que el voseo sigue teniendo una permanencia vital en la ciudad y que está muy generalizado en todos los estratos sociales. Esto contradice la hipótesis que se tenía de que cuanto más se fuera culturizando la población, más desaparecería el voseo (Jang 2013). Existen una serie de factores tales como las actitudes sociales de pertenencia e identidad de los hablantes paisas frente a este fenómeno que asegura la permanencia del voseo en la región por mucho tiempo más. El autor dice que los informantes de su estudio, jóvenes universitarios, tienen un gran sentido de pertenencia al grupo antioqueño del cual hacen parte y por esto el voseo forma parte de su identidad y lo usan con orgullo. Agrega que, para ellos, dejar de usar el vos sería casi como negar su identidad. En el tema de identidad, Weyers (2016) dice que hoy en día hasta es posible advertir un cambio del rol del voseo que, a pesar de haber sido utilizado tradicionalmente en el discurso oral e informal, está cada vez más presente en la comunicación escrita. Según el autor, este cambio está siendo generado por iniciativas gubernamentales para preservar el sentimiento regionalista entre los habitantes de la ciudad. En su análisis de los mensajes de Facebook de la Alcaldía de Medellín durante sus primeros seis meses de administración, Weyers identifica un incremento en el uso del voseo mientras que el tuteo, que solía ser la forma estándar para los medios de comunicación, presenta una disminución considerable. Señala así a este organismo administrativo de la ciudad como el agente que está introduciendo este cambio lingüístico y advierte que dicho cambio resulta en un fortalecimiento del prestigio hacia el voseo en el medio escrito que obedece a la transformación que ha tenido la ciudad en los últimos años.

Los estudios y artículos aquí presentados esbozan un panorama de la situación del fenómeno del voseo en la ciudad de Medellín. No obstante, muchos fueron realizados con la población joven universitaria razón por la cual el presente estudio se concentró, entonces, en identificar los factores sociales que condicionan el voseo y las actitudes de identidad en los hablantes de Medellín teniendo en cuenta hablantes de un rango de edad mucho más amplio; es decir, hablantes de más de una generación. 


\section{Hipótesis de Investigación}

Las hipótesis que orientaron este estudio fueron las siguientes:

(1) Los factores que motivan el uso del voseo en el habla de los habitantes de Medellín estarán relacionados con la edad del interlocutor prediciendo menos uso del vos a mayor edad del interlocutor, el tipo de relación con el interlocutor prediciendo más uso del vos a mayor solidaridad entre los hablantes $y$, finalmente, el género de los hablantes prediciendo que las mujeres tenderán a usar más el tú que los hombres.

(2) Los hablantes paisas tienen una actitud de identidad regional y de pertenencia al grupo antioqueño que motiva el uso del vos en la región sin importar la edad de los hablantes.

\section{Metodología}

\subsection{Participantes}

Todos los informantes que participaron en este estudio son personas paisas que han vivido la mayor parte de su vida en Medellín. Sus edades varían entre los 20 y los 80 años, tienen diferentes niveles de educación y pertenecen a las clases sociales alta y media alta de la ciudad, de estratos 4,5 y 6 . La distribución general de los participantes aparece en la siguiente tabla:

\begin{tabular}{|c|c|c|c|c|}
\hline Edad & clase alta & clase alta & clase alta & clase media alta \\
\hline 20 a 35 años & mujer & hombre & & mujer \\
\hline 36 a 50 años & hombre & hombre & mujer & \\
\hline 51 a 65 años & mujer & & & hombre \\
\hline 66 a 80 años & mujer & hombre & & \\
\hline
\end{tabular}

Tabla 2. Distribución de los participantes. 


\subsection{Cuestionarios}

Para recoger los datos, se utilizaron tres cuestionarios: un cuestionario de información personal, un cuestionario de formas de tratamiento y un cuestionario de actitudes.

\subsubsection{Cuestionario de información personal}

Este cuestionario sirvió como base para entender la información sociodemográfica de los participantes y corroborar su pertenencia al grupo paisa y a las clases sociales relevantes para el estudio. Los participantes respondieron preguntas sobre su edad, su género, su nivel de educación, su clase social y su ocupación. Este cuestionario no fue diseñado para recoger datos que aportaran al análisis de los resultados sino más bien para identificar y controlar el grupo de participantes con las características demográficas necesarias. No obstante, fue de suma importancia para registrar la edad de los participantes y saber si marca una diferencia en el uso del vos.

\subsubsection{Cuestionario de formas de tratamiento}

Este cuestionario de selección múltiple tuvo preguntas apuntadas a identificar los factores que determinan el uso de vos en los hablantes. Los participantes marcaron con una $\mathrm{X}$ en tú, usted, o vos la manera como se dirigen a los diferentes interlocutores presentados. Este cuestionario fue diseñado para responder a la primera pregunta de investigación objeto de este estudio sobre cómo la relación de solidaridad o distancia de los hablantes con su interlocutor y la edad y el género de la persona con la que se habla motivan el uso del vos. Las opciones de interlocutores presentadas en el cuestionario (ver apéndice) incluían interlocutores con los cuales normalmente se tiene una relación estrecha, como los padres, hermanos y amigos cercanos, e interlocutores con los cuales normalmente hay una relación de distancia como una persona que se acaba de conocer, un taxista o una persona que no se conoce muy bien. Además, había interlocutores de edad cercana, como los hermanos y los amigos, y de edad mayor, como señores mayores 
o profesores mayores, para identificar si la edad del interlocutor afecta de alguna manera el uso del vos.

\subsubsection{Cuestionario de actitudes}

Por último, el tercer cuestionario estaba dividido en dos partes y para el presente estudio solamente se utilizaron los datos recogidos en la primera parte del cuestionario que buscaban responder a la segunda pregunta de investigación sobre qué tanto creen los paisas que el voseo está relacionado con su identidad. Esta primera parte recogió datos por medio de enunciados con escala tipo Likert de siete puntos con un rango de respuesta desde 'totalmente en desacuerdo' hasta 'totalmente de acuerdo'. Las preguntas 1 a 5 (apéndice) buscaban establecer si los hablantes opinan que en Medellín el vos está más generalizado que el tú y si consideran que hablar de vos es un rasgo característico de su cultura y de su identidad. Las preguntas 6 a 8, por otro lado, investigaban qué tanto los paisas piensan que el vos está pasando de ser usado solo informalmente para usarse cada vez más en el medio escrito. La segunda parte de este cuestionario incluyó preguntas más abiertas, algunas de tipo sí/no, para recoger información cualitativa que pudiera ser utilizada para triangular los datos en la etapa del análisis de los resultados. Las preguntas abiertas 1, 4 y 5 buscaban complementar la información sobre qué tan generalizado está el voseo en Medellín y las preguntas 2, 3 y 6 sirvieron para triangular información sobre la identidad a la hora de usar el vos y complementar los resultados sobre el tipo de relación, la solidaridad y la permanencia del vos en la región.

\section{Resultados}

Este estudio sobre el uso de vos en Medellín tenía el objetivo de identificar los factores que motivan su uso y determinar si los hablantes paisas creen que el voseo forma parte de su identidad. La primera pregunta de investigación se concentró en tres 
factores, a saber: la edad del interlocutor, el tipo de relación con el interlocutor y el género de los hablantes. Se hipotetizó que a mayor edad de la persona con la que se habla y mayor distancia social menor sería el uso del vos y se predijo que las mujeres tenderían más a utilizar el tú que los hombres. Como se muestra a continuación, los resultados corroboran las hipótesis sobre la edad y la distancia social mas no la hipótesis sobre la distribución del voseo en mujeres y hombres.

\subsection{Edad del interlocutor y tipo de relación}

Como se muestra en la Tabla 3, el voseo es definitivamente utilizado en situaciones de solidaridad y familiaridad.

Factores que motivan el uso de vos

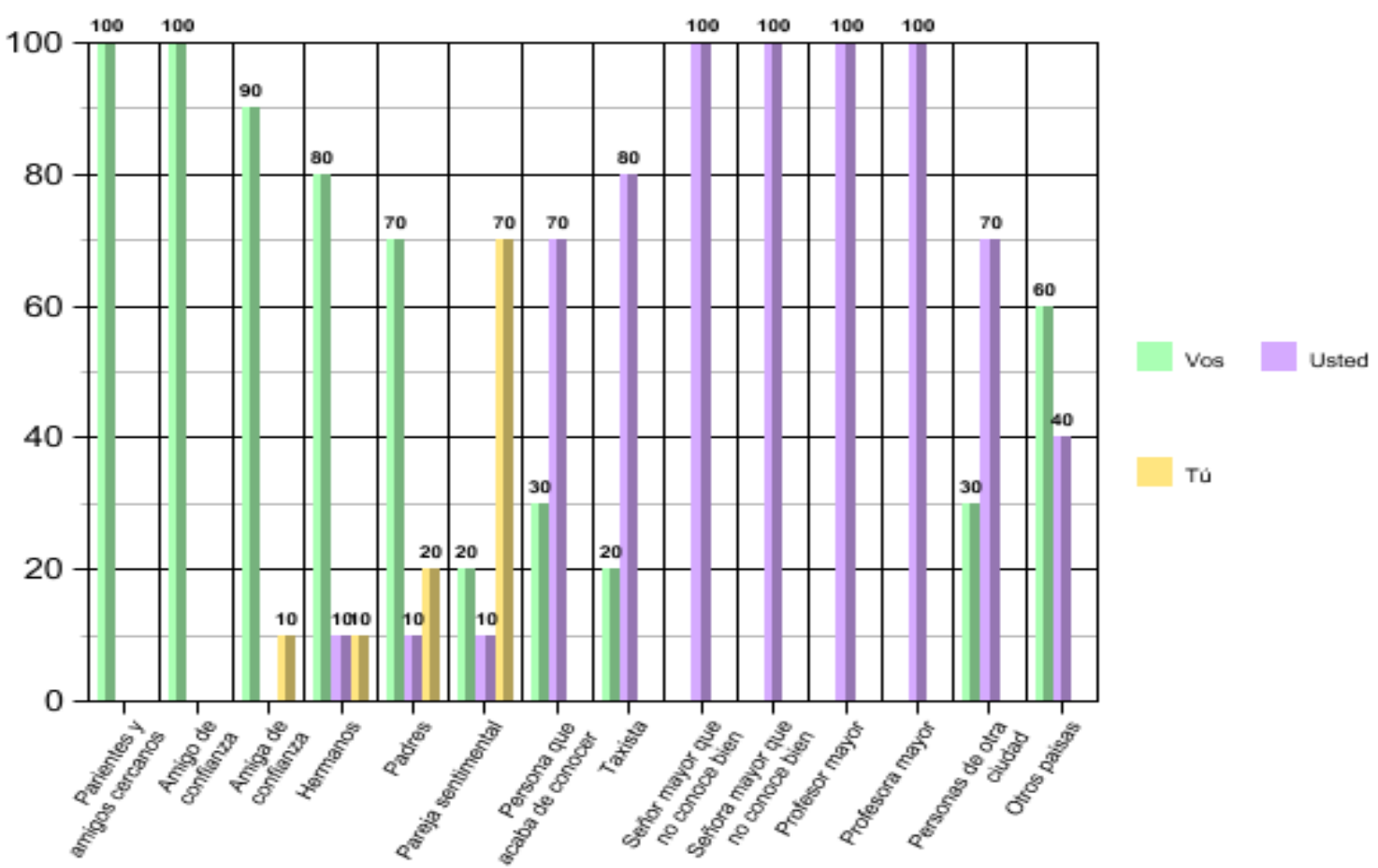

Tabla 3. Factores que motivan el uso de vos.

Para amigos de confianza y parientes cercanos, los hablantes en su totalidad utilizan vos mientras que para personas que no se conocen muy bien, como un taxista o una persona que se acaba de conocer, los hablantes reducen el uso del vos y prefieren, en 
general, utilizar usted. Los resultados presentados en esta tabla muestran un generalizado uso de usted para interlocutores mayores sin importar el tipo de relación. El factor de la edad parece ser determinante a la hora de utilizar usted por encima de vos ya que un señor o una señora que no se conocen bien tienen el mismo porcentaje en el uso de usted que los profesores con los cuales se asume una relación más cercana; esta similitud muy probablemente se debe al hecho de que estos interlocutores son mayores. Así mismo, cuando el interlocutor es una persona que se acaba de conocer, el porcentaje en el uso de usted se reduce a un $70 \%$ para dar paso al uso de vos. Teniendo en cuenta que con una persona que se acaba de conocer el tipo de relación es de alta distancia social y el usted debería ser la norma, la diferencia en porcentajes se debe a que la edad de dicha persona no está especificada en el cuestionario y, por lo tanto, dependiendo de cómo los participantes la imaginen, logran concebir un trato más cercano utilizando vos. Lo mismo sucede con un taxista que en principio es un desconocido y sin embargo algunos participantes lo tratarían de vos debido, tal vez, a la idea de que el taxista puede estar cercano en edad al hablante y motivar un trato más familiar. Parece, entonces, que al estar al frente de una persona que no se conoce o se acaba de conocer, la distancia social que la situación conlleva no es tan relevante como la edad del interlocutor que sí aparenta ser un factor de peso a la hora de escoger la forma de tratamiento. Puede concluirse entonces que usted es el pronombre de distanciamiento estándar a menos que la edad entre los interlocutores se perciba cercana, en cuyo caso los hablantes recurrirán al vos.

En cuanto al trato familiar y solidario, cuando se trata de amigos de confianza y parientes cercanos, todos los hablantes utilizan el vos; sin embargo, cuando se trata de la familia inmediata como padres y hermanos existe cierta variación. Para los padres y hermanos la mayoría de los participantes utiliza vos, aunque logra esbozarse el uso del tú en un porcentaje pequeño. Parece que la preferencia de usar tú en vez de vos para dirigirse a los padres corresponde a una variación individual dado que los dos participantes que utilizan tú con sus padres están bien repartidos en la muestra siendo de género diferente, hombre y mujer, de clase social diferente, él de clase alta y ella de clase media alta, y de dos rangos de edad diferentes. Para dirigirse a los hermanos hay una 
situación similar con ocho participantes prefiriendo el vos, una participante joven tú y una participante mayor usted. Estas dos participantes son mujeres en rangos de edad totalmente opuestos, lo que puede indicar un cambio a través del tiempo en el uso de los pronombres para relacionarse con los hermanos. En cuanto a la pareja sentimental, con la que se asume hay una relación de cercanía, los resultados son variados. El 70 por ciento de los participantes usa tú, el 20 por ciento usa vos y el diez por ciento usted. Los participantes que usan tú en sus relaciones sentimentales, que son la mayoría, están todos en los rangos de edad más bajos. Esto puede significar que los jóvenes están adoptando la forma que es relativamente más nueva en la región y que prefieren usar tú para tratar a sus parejas de manera especial y no el vos que es como tratan a todas las otras personas que son cercanas a ellos.

\subsection{El género de los hablantes}

En cuanto al género de los hablantes, los resultados no confirman la hipótesis de que las mujeres usan más el tú que los hombres. Por el contrario, los datos muestran que para el número total de veces que se reportó el uso de tú en todo el estudio, un 55 por ciento corresponde a los hombres y un 45 por ciento a las mujeres. Los resultados de este estudio en particular, donde los hombres hacen más uso del tú que las mujeres, también contradicen estudios anteriores que indican que las mujeres, por ser las que tienden a usar las formas de tratamiento consideradas estándar, presentan más variedad en su habla y una tendencia mayor a utilizar tú (Millán 2011) y estudios que dicen que las mujeres tienden a tutear más que los hombres porque el tú tiene un matiz feminizante (Jang 2015).

\subsection{La identidad}

A la segunda pregunta de investigación sobre si los hablantes paisas tienen una actitud de identidad regional y de pertenencia al grupo antioqueño que motiva el uso del vos en la región, los resultados son contundentes. En la Tabla 4 se ven los porcentajes de 
favorabilidad de las respuestas de los participantes a las diferentes preguntas sobre el tema (preguntas 1 a 5 , apéndice).

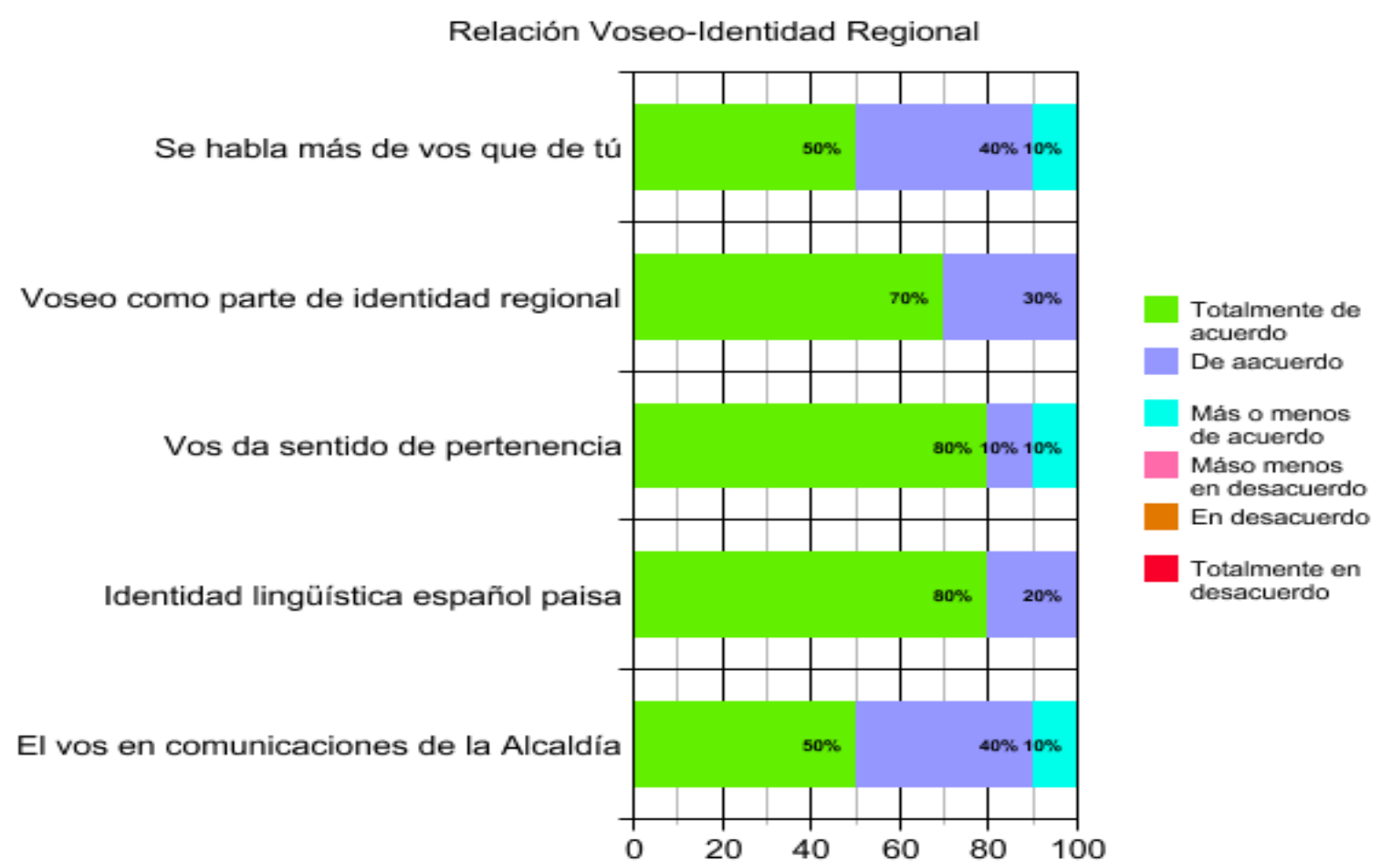

Tabla 4. Relación del voseo con la identidad regional paisa.

Por ser un cuestionario con escala de 1 a 7 puntos y haber 10 participantes, cada respuesta cuenta con un total máximo de 70 puntos. Se sumaron los puntos que cada participante dio a cada pregunta y la suma se convirtió a cantidades porcentuales que indican, en última instancia, qué tanto están los participantes de acuerdo con las preguntas. Las respuestas de todos los participantes estuvieron por encima de la media de cuatro puntos en todas las categorías; es decir, no hay resultados que indiquen que los participantes están en desacuerdo. Lo interesante de este hallazgo es que esto es cierto para todas las generaciones estudiadas. Hablantes desde los 20 hasta los 80 años coinciden en esta tendencia. En general, los paisas reconocen que en Medellín se habla más de vos que de tú, lo que corrobora los resultados de Millán (2011) y que el voseo hace parte de la identidad lingüística del español paisa y que les da sentido de pertenencia al grupo antioqueño. Esta relación del voseo con la identidad regional fue 
triangulada cualitativamente con las preguntas abiertas de la segunda parte del cuestionario de actitudes. Por ejemplo, a la pregunta de si seguirían hablando de vos al visitar otra ciudad o si hablarían con sus amigos bogotanos de tú, el 80 por ciento dijo que seguiría hablando de vos y las razones, inequívocamente, apuntan a la relación que perciben entre el voseo y su identidad como paisas. Comentarios del tipo "hablaría de vos porque me da identidad paisa" o "nuestro trato de vos está muy arraigado en nuestra cultura" son ejemplos de esta percepción. Más aún, "hablaría de vos porque el hecho que me encuentre en otra ciudad no quiere decir que deje de ser paisa" indica que, para esta participante, hablar de vos es, sin lugar a dudas, sinónimo absoluto de ser paisa. El 20 por ciento que no vosearía tiene motivos de acomodación lingüística que valdría la pena analizar en futuros estudios. La preferencia en este caso sería usted ya que "en Bogotá no se usa el vos; vos es de Antioquia". Como puede verse, la relación del vos con la identidad paisa está presente, pero estos hablantes prefieren acomodarse a sus interlocutores bogotanos y utilizar el pronombre de distanciamiento para referirse a ellos.

\section{Discusión}

Este estudio es relevante en el campo de la variación lingüística, más específicamente en la variedad del español latinoamericano y es importante porque, a diferencia de otros países donde el voseo es la norma a nivel nacional, en Medellín los hablantes tienen la posibilidad de utilizar otras opciones como tú y usted y a pesar de esto el voseo sigue vigente. Esto se debe, como aquí se muestra, al profundo sentimiento de identidad regional que caracteriza a los hablantes paisas, lo que significa que esta característica de diferenciación dialectal está lejos de desaparecer. En términos generales, los paisas utilizan usted como pronombre de distanciamiento cuando el interlocutor es mayor o cuando no se tiene una relación estrecha con él. Para tratamiento de confianza, existen dos opciones, tú y vos, que conviven día a día en el discurso de los hablantes. Sin embargo, éstos prefieren y usan mucho más el vos que el tú y están conscientes de que lo hacen porque es un distintivo de identidad regional. 
En los resultados aquí presentados se observaron tendencias de las generaciones más jóvenes a utilizar tú con su pareja sentimental lo que puede ser indicio de un cambio lingüístico en progreso y de la manera como el tú está permeando poco a poco la sociedad antioqueña.

\section{Conclusión}

El fenómeno del voseo es un fenómeno con mucho por explorar. Futuros estudios del voseo en Medellín deberán concentrarse en el posible cambio lingüístico que se está llevando a cabo en la comunicación escrita de la ciudad y en la percepción que los hablantes tienen de este cambio en relación con su identidad. Otro tema a explorar es la idea de acomodación lingüística que puede motivar a los hablantes paisas a utilizar una forma de tratamiento pronominal diferente del vos con interlocutores de otras regiones del país y cómo dicha acomodación está ligada a actitudes que los hablantes tienen hacia estos otros grupos sociales. Más aún, también debería ser explorada la medida en que formas relativamente nuevas como el tú están permeando la cultura antioqueña y su incidencia en las diferentes clases sociales.

Por último, este estudio ha mostrado que el fenómeno del voseo está ligado a la identidad de los hablantes. Si el voseo está estrechamente ligado a la identidad regional de una ciudad como Medellín en un país que no es voseante a nivel nacional, debe estar ligado también a la identidad de los hablantes de países donde se usa de manera general. Esto asegura la permanencia y relevancia del fenómeno a nivel del continente americano lo que lleva a pensar que tal vez es hora de comenzar a enseñarlo en las aulas de español como lengua extranjera. Si bien es cierto que los aprendices no necesitan aprender el voseo para hablar un buen español, también es cierto que deberían aprender a reconocer esta característica dialectal para verdaderamente entender el idioma como es hablado por millones de personas en el continente. Por lo tanto, este estudio es una contribución a los campos de la dialectología y de la sociolingüística hispánicas para propiciar diálogos 
en el campo de la enseñanza de nuestra lengua, así como en los ámbitos donde se hable español como lengua nativa.

\section{Referencias}

ANGULO, Lizandro (2009) "Voseo, el otro castellano de América", Revista de Historia de la Educación Latinoamericana, 14, 267-288.

BENAVIDES, Carlos (2003) "La distribución del voseo en Hispanoamérica”, Hispania, 86(3), 612-623.

JANG, Ji Son (2013) "Voseo medellinense como expresión de identidad paisa", Íkala, Revista de Lenguaje y Cultura, 18(1), 61-81.

JANG, Ji Son (2015) "Matiz feminizante del tuteo y el futuro del voseo en el departamento de Antioquia (Colombia)", Estudios Filológicos, 56, 85-99.

KAPOVIC, Marco (2007) "Fórmulas de tratamiento en dialectos de español; fenómenos de voseo y ustedeo", Hieronymous, 1. Retrieved April 6, 2015, from

<http://www.unizd.hr/portals/43/broj_1_2007/marko_kapovic_voseo_y_ustedeo.pdf>

MILLÁN, Mónica (2011) Pronouns of address in informal contexts: a comparison of two dialects of Colombian Spanish. (Doctoral dissertation. University of Illinois at Urbana-Champaign). Retrieved Retrieved April 13, 2015, from

<https://www.ideals.illinois.edu/bitstream/handle/2142/29846/Millan_Monica.pdf?sequence=1>

VILLEGAS, Francisco (1963), "The voseo in Costa Rican Spanish", Hispania, 46(3), 612-615.

WEYERS, Joseph (2016) "Medellín cuenta con vos: El papel cambiante de voseo en la comunicación escrita", Comunicación, 35, 67-81. 
Dialectologia 24 (2020), 91-109.

ISSN: 2013-2247

Anexo 1

Cuestionario de Formas de Tratamiento

\section{CUESTIONARIO SOBRE FORMAS DE TRATAMIENTO - VOS / TÚ / USTED}

En general, cómo trataría a cada una de estas personas: ¿de vos, tú o usted?

\begin{tabular}{|c|c|c|c|c|}
\hline & Tú & Vos & Usted & No sé \\
\hline \multicolumn{5}{|l|}{ Sus padres } \\
\hline \multicolumn{5}{|l|}{ Sus hermanos } \\
\hline \multicolumn{5}{|l|}{ Su pareja sentimental } \\
\hline \multicolumn{5}{|l|}{ Una amiga de confianza } \\
\hline \multicolumn{5}{|l|}{ Un amigo de confianza } \\
\hline \multicolumn{5}{|l|}{ Sus parientes y amigos muy cercanos } \\
\hline \multicolumn{5}{|l|}{ Una persona que acaba de conocer } \\
\hline \multicolumn{5}{|l|}{ Un señor mayor que no conoce } \\
\hline \multicolumn{5}{|l|}{ Una señora mayor que no conoce } \\
\hline \multicolumn{5}{|l|}{ Un taxista } \\
\hline \multicolumn{5}{|l|}{ Una profesora mayor } \\
\hline \multicolumn{5}{|l|}{ Un profesor mayor } \\
\hline \multicolumn{5}{|l|}{ Una persona de otra ciudad, por ejemplo, Bogotá } \\
\hline Otras personas paisas en general & & & & \\
\hline
\end{tabular}

Anexo 2

Cuestionario de Actitudes

CUESTIONARIO SOBRE LAS ACTITUDES DE LOS PAISAS HACIA EL VOSEO

I. Por favor marque con una $\mathrm{X}$ qué tanto está usted de acuerdo o en desacuerdo con los siguientes enunciados.

1. En términos generales, en Medellín hablamos más de vos que de tú.

\begin{tabular}{|c|c|c|c|c|c|c|}
\hline $\begin{array}{c}\text { totalmente en } \\
\text { desacuerdo }\end{array}$ & $\begin{array}{c}\text { en } \\
\text { desacuerdo }\end{array}$ & $\begin{array}{c}\text { más o menos } \\
\text { en } \\
\text { desacuerdo }\end{array}$ & neutral & $\begin{array}{c}\text { más o } \\
\text { menos de } \\
\text { acuerdo }\end{array}$ & de acuerdo & $\begin{array}{c}\text { totalmente de } \\
\text { acuerdo }\end{array}$ \\
\hline & & & & & \\
\hline
\end{tabular}

2. El voseo forma parte de la identidad regional de los antioqueños.

\begin{tabular}{|c|c|c|c|c|c|c|}
\hline $\begin{array}{c}\text { totalmente en } \\
\text { desacuerdo }\end{array}$ & $\begin{array}{c}\text { en } \\
\text { desacuerdo }\end{array}$ & $\begin{array}{c}\text { más o menos } \\
\text { en } \\
\text { desacuerdo }\end{array}$ & neutral & $\begin{array}{c}\text { más o } \\
\text { menos de } \\
\text { acuerdo }\end{array}$ & de acuerdo & $\begin{array}{c}\text { totalmente de } \\
\text { acuerdo }\end{array}$ \\
\hline & & & & & & \\
\hline
\end{tabular}

3. Hablar de vos le da a usted sentido de pertenencia a la cultura paisa. 


\begin{tabular}{|c|c|c|c|c|c|c|}
\hline $\begin{array}{l}\text { totalmente en } \\
\text { desacuerdo }\end{array}$ & $\begin{array}{c}\text { en } \\
\text { desacuerdo }\end{array}$ & $\begin{array}{c}\text { más o menos } \\
\text { en } \\
\text { desacuerdo }\end{array}$ & neutral & $\begin{array}{c}\text { más o } \\
\text { menos de } \\
\text { acuerdo }\end{array}$ & de acuerdo & $\begin{array}{l}\text { totalmente de } \\
\text { acuerdo }\end{array}$ \\
\hline \multicolumn{7}{|c|}{ 4. El vos es un símbolo de la identidad lingüística del español paisa. } \\
\hline $\begin{array}{l}\text { totalmente en } \\
\text { desacuerdo }\end{array}$ & $\begin{array}{c}\text { en } \\
\text { desacuerdo }\end{array}$ & $\begin{array}{c}\text { más o menos } \\
\text { en } \\
\text { desacuerdo }\end{array}$ & neutral & $\begin{array}{c}\text { más o } \\
\text { menos de } \\
\text { acuerdo }\end{array}$ & de acuerdo & $\begin{array}{l}\text { totalmente de } \\
\text { acuerdo }\end{array}$ \\
\hline & & & & & & \\
\hline
\end{tabular}

5. Cuando la Alcaldía de Medellín o los canales regionales utilizan el vos en sus comunicaciones, esto aumenta el sentido de pertenencia de los paisas con su ciudad.

\begin{tabular}{|c|c|c|c|c|c|c|}
\hline $\begin{array}{c}\text { totalmente en } \\
\text { desacuerdo }\end{array}$ & $\begin{array}{c}\text { en } \\
\text { desacuerdo }\end{array}$ & $\begin{array}{c}\text { más o menos } \\
\text { en } \\
\text { desacuerdo }\end{array}$ & neutral & $\begin{array}{c}\text { más o } \\
\text { menos de } \\
\text { acuerdo }\end{array}$ & $\begin{array}{c}\text { de acuerdo } \\
\text { totalmente de } \\
\text { acuerdo }\end{array}$ \\
\hline & & & & & & \\
\hline
\end{tabular}

6. El vos va a seguir siendo parte de la cultura paisa.

\begin{tabular}{|c|c|c|c|c|c|c|}
\hline $\begin{array}{c}\text { totalmente en } \\
\text { desacuerdo }\end{array}$ & $\begin{array}{c}\text { en } \\
\text { desacuerdo }\end{array}$ & $\begin{array}{c}\text { más o menos } \\
\text { en } \\
\text { desacuerdo }\end{array}$ & neutral & $\begin{array}{c}\text { más o } \\
\text { menos de } \\
\text { acuerdo }\end{array}$ & de acuerdo & $\begin{array}{c}\text { totalmente de } \\
\text { acuerdo }\end{array}$ \\
\hline & & & & & & \\
\hline
\end{tabular}

7. En la televisión regional se está usando el vos cada vez con más frecuencia.

\begin{tabular}{|c|c|c|c|c|c|c|}
\hline $\begin{array}{c}\text { totalmente en } \\
\text { desacuerdo }\end{array}$ & $\begin{array}{c}\text { en } \\
\text { desacuerdo }\end{array}$ & $\begin{array}{c}\text { más o menos } \\
\text { en } \\
\text { desacuerdo }\end{array}$ & neutral & $\begin{array}{c}\text { más o } \\
\text { menos de } \\
\text { acuerdo }\end{array}$ & de acuerdo & $\begin{array}{c}\text { totalmente de } \\
\text { acuerdo }\end{array}$ \\
\hline & & & & & & \\
\hline
\end{tabular}

8. El vos ha pasado de ser solo oral y aparece ahora en la comunicación escrita de la ciudad (como anuncios en la calle, en el metro, en el periódico, etc.)

\begin{tabular}{|c|c|c|c|c|c|c|}
\hline $\begin{array}{c}\text { totalmente en } \\
\text { desacuerdo }\end{array}$ & $\begin{array}{c}\text { en } \\
\text { desacuerdo }\end{array}$ & $\begin{array}{c}\text { más o menos } \\
\text { en } \\
\text { desacuerdo }\end{array}$ & neutral & $\begin{array}{c}\text { más o } \\
\text { menos de } \\
\text { acuerdo }\end{array}$ & $\begin{array}{c}\text { de acuerdo } \\
\text { totalmente de } \\
\text { acuerdo }\end{array}$ \\
\hline & & & & & & \\
\hline
\end{tabular}

II. Por favor responda a las siguientes preguntas de la mejor manera que pueda, basado en lo que usted sabe o piensa. Si no sabe, responda "no sé". Si tiene comentarios adicionales, por favor escríbalos al final del cuestionario.

1. ¿Por qué cree que los paisas hablan de vos cuando en otras regiones del país sólo se utiliza tú o usted?

2. ¿Le parece raro que una persona que acaba de conocer lo trate de vos? Sí __ No ¿Por qué?

3. ¿Si estuviera en Bogotá, hablaría de tú con sus amigos bogotanos o seguiría hablando de vos? Tú ___ Vos

¿Por qué? 
Dialectologia 24 (2020), 91-109.

ISSN: 2013-2247

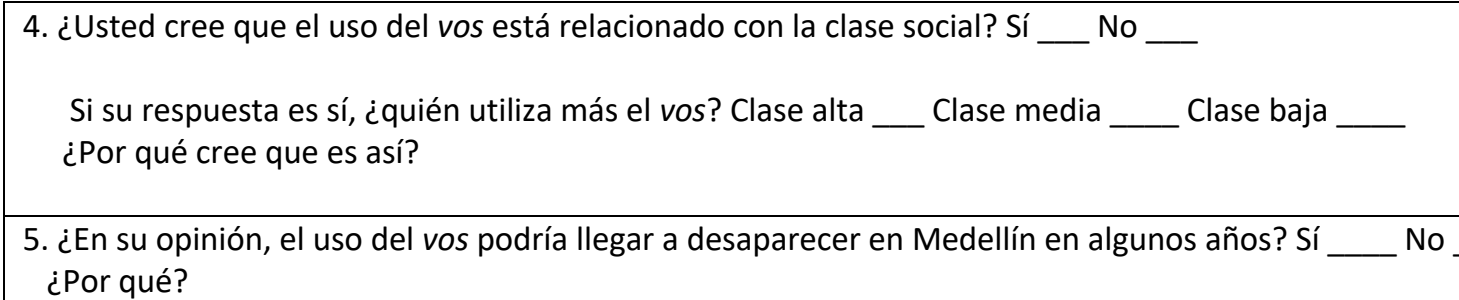

6. En general, ¿qué piensa usted de los paisas? ¿Cómo los compara con otros grupos del país?

Comentarios adicionales: 